3476 patients were treated. Incidence of any adverse events (AEs), serious AEs and investigator-defined drug-related AEs was similar across treatment groups within each trial. AEs reported by $\geq 5 \%$ of patients were similar across all treatment groups within each trial (Table). The number of cardiovascular AEs was small in all five studies and comparable between tiotropium Respimat ${ }^{\circledR}$ and placebo. No deaths occurred in any trial. Conclusion: Once-daily tiotropium Respimat ${ }^{\circledR}$ is well tolerated and comparable with placebo in adult patients with symptomatic asthma receiving at least low- to high-dose ICS.

\section{P232 TREATMENT OF ALLERGIC RHINITIS WITH THEOPHYLLINE : A DOUBLE-BLIND, RANDOMISED, CROSSOVER STUDY}

P Sankaran, C Brockwell, A Clark, AM Wilson. University of East Anglia, Norwich, UK

\subsection{6/thoraxjnl-2014-206260.360}

Background Allergic rhinitis and Asthma are considered as 'one airway disease'. Theophylline has been used as a bronchodilator in asthma for decades but more recently its anti-inflammatory properties have been identified. We hypothesise that treatment with low dose theophylline in patients with persistent allergic rhinitis is likely to improve the total nasal symptom scores and there by demonstrate a clinically meaningful difference.

Methods This was a single centre double- blind, randomised, placebo-controlled cross-over study of the effects of theophylline (one capsule of Theophylline $200 \mathrm{mgs}$ as Uniphyllin continus twice a day for 4 weeks) in 21 patients with persistent allergic rhinitis in Norwich, U. K. Reference: NCT0113278. Primary outcome was Total Nasal Symptom Score (TNSS) after each intervention period. Secondary endpoint measures were differences in the domiciliary average total nasal symptom score, differences in nasal peak inspiratory flow (PNIF), differences in domiciliary nasal peak inspiratory flow and difference in SinoNasal Outcome Test (SNOT)-22.

Results Primary Endpoint

There was no significant $(p=0.276)$ difference in Total Nasal Symptoms scores during Theophylline treatment period and placebo period, mean (SD) (Table). The intention-to-treat analysis results were in keeping with the per protocol analysis.

Secondary End points

PNIF in the Theophylline period was $112.38( \pm 43.49)$ compared to the placebo period $122.86( \pm 53.77), \mathrm{p}=0.171$ (Table). There was no change in SNOT-22 $(\mathrm{p}=0.867)$ between treatment periods but there was a non-significant improvement with Theophylline $(39.00 \pm 19.78)$ compared to placebo $(38.00 \pm$ 19.63) treatment period. There was a non-significant improvement in the domiciliary total nasal symptom scores (TNSS) between Theophylline $(3.53 \pm 2.35)$ and placebo $(2.81 \pm 2.46)$. Nasal scrape samples were stained with HDAC2 antibodies and the signals were very week.

\begin{tabular}{|c|c|c|c|c|}
\hline Outcome Variable & $\begin{array}{l}\text { Placebo } \\
\text { Mean (SD) }\end{array}$ & $\begin{array}{l}\text { Theophylline } \\
\text { Mean (SD) }\end{array}$ & $\mathrm{p}$ Value & $95 \% \mathrm{Cl}$ \\
\hline TNSS & $4.90(3.08)$ & $4.14(2.33)$ & 0.276 & $-0.76(-2.13,0.61)$ \\
\hline PNIF & $122.86(53.77)$ & $112.38(43.49)$ & 0.171 & $-10.48(-25.49,4.54)$ \\
\hline SNOT-22 & $38.00(19.63)$ & 39.00 (19.78) & 0.867 & $0.63(-6.67,7.92)$ \\
\hline
\end{tabular}

Conclusion This is the first study evaluating Theophylline in persistent rhinitis. Low-dose Theophylline had no significant effects on Total nasal Symptom scores; Rhinosinusitis symptoms and nasal patency assessed using peak nasal inspiratory flow. There was a non-significant improvement in the total nasal symptom scores and sino-nasal outcome test and domiciliary nasal scores.

\section{P233 LONG-TERM IMPACT OF INHALED CORTICOSTEROIDS ON BONE MINERAL DENSITY AND FRACTURE RISK IN PATIENTS WITH ASTHMA: SYSTEMATIC REVIEW AND META-ANALYSIS}

YK Loke, D Gilbert, M Thavarajah, P Blanco, AM Wilson. University of East Anglia, Norwich, UK

\subsection{6/thoraxjnl-2014-206260.361}

Background A recent meta-analysis of 16 randomised controlled trials (RCTs) and 7 observational studies demonstrated a modest but statistically significant increase in fracture risk with inhaled corticosteroid (ICS) use in chronic obstructive pulmonary disease. However, it is not clear whether ICS use has similar skeletal adverse effects in patients with asthma. We aimed to evaluate the association between ICS and fractures and changes in bone mineral density when used for $>12$ months in asthma.

Methods We initially searched MEDLINE and EMBASE in July 2013, and performed an updated PubMed search in June 2014. We used a combination of search terms involving drug name and adverse effects of interest, and we also hand-searched reference lists of existing systematic reviews and trial reports. We selected RCTs and controlled observational studies of any ICS vs nonICS control treatment for asthma (at least 52 weeks duration). Meta-analysis of odds ratios was conducted using RevMan 5.3 with the primary outcome measure being fracture events. We also analysed mean differences in bone mineral density (gram per $\mathrm{cm}$ squared) using inverse variance method. Heterogeneity was assessed using the I2 statistic.

Results We selected nine RCTs and 11 observational studies for the meta-analysis. There was no significant association between ICS and fractures in children in one RCT, or in a pooled analysis of two observational studies, (OR 1.02, 95\% CI 0.94-1.10). No significant fracture risk in adults was reported in 4 observational studies (pooled OR 1.09, 95\% CI 0.45-2.62). Meta-analysis of bone mineral density at the lumbar spine did not show significant reductions with ICS use in children (three RCTs and three observational studies), or in adults (three RCTs and four observational studies). Similarly, meta-analysis of bone mineral density at the neck of femur in adults did not demonstrate significant reductions compared to control (three RCTs and four observational studies).

Conclusion In our systematic review of 20 studies, use of ICS for $>12$ months in patients with asthma was not associated with statistically significant adverse effects on bone mineral density or fractures.

\section{P234 IMPACT OF INHALED CORTICOSTEROIDS ON GROWTH IN CHILDREN WITH ASTHMA: SYSTEMATIC REVIEW AND META-ANALYSIS}

YK Loke, M Thavarajah, P Blanco, AM Wilson. University of East Anglia, Norwich, UK

10.1136/thoraxjnl-2014-206260.362 